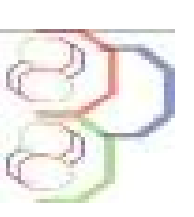

\title{
Diversité Génétique des Premières Variétés de Canne à Sucre d'Origine Réunionno-Ivoirienne Présélectionnées à Ferké en Côte d'Ivoire
}

\author{
YYM Béhou¹2, CB Péné1, A Zézé2, JD Oura1, Y Ouattara1, M Boua1 \\ 1 Direction Recherche et Développement, SUCAF-CI/SOMDIAA, 01 BP 1967 Abidjan, Côte d'Ivoire. \\ 2 UMRI : Sciences Agronomiques et Génie Rural, EDP/INPHB, BP 1313 Yamoussoukro, Côte d'Ivoire. \\ Corresponding Author Email: mbehou@sucafci.somdiaa.com
}

Original submitted in on $12^{\text {th }}$ March 2018. Published online at www.m.elewa.org on $31^{\text {st }}$ May 2018 https://dx.doi.org/10.4314/jab.v125i1.8

\section{RÉSUMÉ}

Objectifs : L'étude visait à contribuer à l'amélioration de la productivité en culture cannière en Côte d'Ivoire. Plus spécifiquement, elle visait à caractériser la diversité génétique du tout premier lot de 148 variétés de canne à sucre d'origine réunionno-ivoiriennes présélectionnées au stade " une ligne » en vue d'essais classiques de sélection de 1 ère et 2 ème étapes à conduire par la suite dans les conditions d'exploitation des complexes sucriers de Ferké.

Méthodes et résultats : Elle a été conduite en station expérimentale, sous irrigation par aspersion en couverture intégrale, au complexe sucrier de Ferké 2, au Nord Côte d'Ivoire. Ces variétés ont été présélectionnées en 1 ère repousse parmi 985 clones plantés par famille, à raison d'un individu par ligne de $3 \mathrm{~m}$ de long sans répétition et comparés à une variété témoin (SP70-1006). Celle-ci a été plantée régulièrement toutes les 5 lignes afin de faciliter la notation des clones pour différents caractères agromorphologiques et phytosanitaires par comparaison avec le témoin. Les variables quantitatives et qualitatives relatives aux variétés présélectionnées, observées à la récolte en 1 ère repousse à l'âge de 10 mois, ont fait l'objet d'une série d'analyses multivariées. L'étude a montré que les variables quantitatives permettant de mieux expliquer la diversité des variétés présélectionnées comprenaient, dans l'ordre décroissant, le taux de floraison, le nombre de tiges $/ 3 \mathrm{~m}$, le diamètre des tiges et la longueur des tiges. Ces variables ont fortement contribué à la discrimination de 8 groupes qui suggéraient une assez bonne diversité génétique parmi les 148 clones présélectionnés. Les 4 groupes les mieux représentés $(63,5 \%$ de l'effectif total) comprenaient chacun 20 à 30 individus, tandis que les moins représentés (36.5\%) 11 à 15 individus chacun.

Conclusion et application des résultats : Les variables qualitatives les plus pertinentes dans la discrimination des groupes portent sur très bon tallage, tallage moyen, port plus ou moins droit, d'une part, et port droit, d'autre part, qui sont associés, respectivement, aux groupes G1, G7 et G2. Quant aux variables port penché et port en bataille qui sont également pertinentes, elles semblaient être associées à 6 groupes à la fois (G2, G3, G4, G5, G6, G8). La variabilité génétique ainsi révélée est nécessaire aux prochains essais de sélection prévus en plantations commerciales.

Mots clés. Sélection massale, vigueur végétative, stade ligne, variable quantitative, variable qualitative, analyse multivariée, groupe dissemblable, adaptation, contexte agro-écologique. 


\section{ABSTRACT \\ Genetic Diversity of First Sugarcane Varieties of Reunion-Ivorian Origin Preselected in Ferké, Ivory Coast}

Objectives: The overall study objective was to contribute to sugarcane yield improvement in Ivory Coast. It aimed to characterize the genetic diversity of the first batch of 148 sugar cane varieties of Reunion-Ivorian origin preselected at the "one-row" stage for standard selection trials of 1st and 2nd stages to be carried out under Ferké commercial field conditions.

Methodology and results: It was conducted on Ferké 2 experimental station under full sprinkler irrigation in northern Ivory Coast. These varieties were pre-selected in 1st regrowth among 985 clones planted per family, one individual per row $3 \mathrm{~m}$ long without repetition and compared to a control variety (SP701006). That one was replicated several times every 5 rows to ease agronomics observations of clones in comparison with the control. Quantitative as well as qualitative traits observed at the age of 10 months were subjected to a series of multivariate analyses. The study showed that quantitative traits, which better explained diversity of varieties, were the following in decreasing order: flowering rate, number of millable stalks/3m, stalk diameter, and stalk height. Those phenotypic traits highly contributed to discrimination of varieties into 8 clusters, which suggested a good genetic diversity among all 148 preselected clones. Four best-represented clusters (63.5\% of total) comprised 20-30 individuals each, whereas less represented clusters (36.5\%) involved 11-15 individuals each.

Conclusion and application of results: Quantitative traits most relevant in variety clustering were high tillering, moderate tillering, erect traits, which were associated respectively with clusters G1, G7 and G2. As for lodging traits, they were associated with 6 clusters all together, namely G2, G3, G4, G5, G6 and G8. The genetic variability so shown is a prerequisite for further selections trials under commercial field conditions.

Keywords: visual selection, vegetative vigor, one-row stage, phenotypic trait, multivariate analysis, clustering, adaptation, agro-ecologic condition.

\section{INTRODUCTION}

La canne à sucre est cultivée industriellement en Côte d'Ivoire depuis 1970. La productivité agricole du secteur sucrier ivoirien, jusqu'en 2010, était limitée à 8 tonnes de sucre à l'hectare (Péné et Tuo, 2007 ; Péné et al, 2010). Le matériel végétal, d'origine diversifiée, qui est cultivé en Côte d'Ivoire et dans la plupart des pays en Afrique de l'Ouest et du Centre a été introduit à l'état de boutures de variétés commerciales, de variétés élites ou de variétés présélectionnées à partir de la quarantaine du CIRAD à Montpellier. Généralement, il a été sélectionné dans ces pays de destination pour son adaptation aux conditions pédoclimatiques et phytosanitaires locales. Dans le cas des complexes sucriers de Ferké, en Côte d'Ivoire, l'exploitation de nouvelles variétés plus productives en sucre (rendement moyen de 9 à 10 tonnes à l'hectare) a contribué notablement à l'amélioration de la productivité agricole d'environ $25 \%$ de 2010-11 à 2014-15 (Péné et al, 2016).
Malgré cette amélioration substantielle, la production nationale de sucre reste encore déficitaire avec environ 200 mille t/an pour un besoin de consommation estimé à 240 mille tan (Zadi et al, 2017). Le foreur de tiges Eldana saccharina et deux maladies endémiques économiquement importantes, en l'occurrence le charbon et l'échaudure foliaire causés respectivement par Ustilago scitaminea et Xanthomonas albilieans, constituent les principales contraintes biotiques dans les périmètres sucriers ivoiriens (Kouamé et al, 2010, Zadi et al, 2017). Ces contraintes biotiques comptent de plus en plus parmi les facteurs limitants de la productivité agricole avec une perte de rendements en cannes et en sucre pouvant atteindre parfois 20 voire $30 \%$ due notamment au foreur de tiges (Péné et al, 2016). Pour limiter l'incidence de ces maladies et du ravageur, la sélection variétale a été privilégiée en Côte d'Ivoire depuis au moins cinq décennies. 
Cependant, dans le cadre de cet effort de sélection, le matériel végétal introduit dans le pays jusqu'en 2013-14 offrait une diversité génétique relativement étroite avec par conséquent une capacité d'adaptation limitée (Péné et Déa, 2000). L'introduction, chaque année, d'un nombre élevé de semences hybrides de canne à sucre issues de différents croisements biparentaux (environ 8000 graines/an) permet d'assurer une plus grande variabilité génétique au sein du matériel végétal (seedlings et clones) à sélectionner. La sélection à partir de seedlings puis de clones de canne à sucre produits localement, permet donc d'accroître les chances de déterminer à terme de nouvelles variétés répondant à des défis multiformes tels que: productivité en sucre élevée, bonne

\section{MATÉRIEL ET MÉTHODES}

Site d'étude : L'étude a été menée à la station expérimentale du complexe sucrier de Ferké 2 , au Nord Côte d'Ivoire $\left(9^{\circ} 20^{\prime}-9^{\circ} 60^{\prime} \mathrm{N}, 5^{\circ} 22^{\prime}-5^{\circ} 40^{\prime} \mathrm{O}, 325 \mathrm{~m}\right)$. Le climat qui prévaut dans la zone est de type tropical sec caractérisé par deux saisons : l'une sèche, s'étend de novembre à avril et l'autre humide, de mai à octobre. La saison sèche est marquée par l'harmattan, un vent chaud et sec qui s'étend de mi-novembre à fin janvier. Le régime pluviométrique est de type unimodal, centré sur les mois d'août-septembre qui cumulent presque la moitié de la hauteur moyenne annuelle de précipitations égale à $1200 \mathrm{~mm}$ avec une température moyenne journalière de $27{ }^{\circ} \mathrm{C}$. Les températures minimale et maximale moyennes journalières sont respectivement de 32,5 et $21^{\circ} \mathrm{C}$. Pour satisfaire les besoins en eau de la canne à sucre, les apports par irrigation avoisinent les $700 \mathrm{~mm}$ par an (Péné et al, 2010). Le périmètre sucrier renferme majoritairement des sols peu profonds, formé sur granite ou gneiss. Les principales unités de sol sont de types ferralitique, hydromorphe de bas-fond et sableux des terrasses du fleuve Bandama. La texture du sol de la parcelle d'essai est sablo-argileuse.

Matériel végétal : Le matériel végétal présélectionné en 1ère repousse au stade ligne parmi 985 clones plantés par famille et comparés à un témoin d'origine brésilienne (SP70-1006), comprenait 148 variétés d'origine réunionno-ivoirienne $(\mathrm{RCl})$. Ces variétés sont issues du 1er lot de semences hybrides de canne à sucre importées en 2014 de La Réunion auprès du Centre Technique Sucrier (eRcane). Ces semences adaptation aux conditions agro-écologiques locales, richesse en fibre élevée $(13-16 \% \mathrm{C})$ pour la production de bagasse utilisée comme biocombustible dans la production d'électricité nécessaire à l'usinage de canne et au pompage de l'eau d'irrigation, tallage élevé avec couverture rapide du sol pour limiter naturellement l'enherbement tout en réduisant significativement les coûts liés au contrôle de celui-ci et port végétatif érigé avec épaillage facile pour minimiser les pertes de rendements et le non-canne en récolte mécanisée. La présente étude vise à caractériser la variabilité génétique des premières variétés de canne à sucre d'origine Réunionnoivoirienne présélectionnées localement en vue du stade de la 4ème étape de sélection variétale.

sont issues de croisements bi-parentaux de variétés commerciales ou élites d'origines diversifiées (Réunion, Brésil, Australie, Soudan, Floride, Colombie, Afrique du Sud).

Dispositif expérimental : Le dispositif expérimental adopté au stade ligne était un bloc incomplet, non randomisé comportant 985 clones plantés chacun sur une ligne de $3 \mathrm{~m}$ sans répétition excepté la variété témoin. Les clones, répartis en 60 familles, ainsi que le témoin ont été plantés en lignes simples (équidistantes de $1.5 \mathrm{~m}$ ) en novembre 2015 sur 17 blocs de $7 \mathrm{~m}$ de largeur et $30 \mathrm{~m}$ de longueur. Pour permettre une comparaison aisée des clones avec le témoin, celui-ci a été planté régulièrement tous les $7.5 \mathrm{~m}$ (après 5 individus). Pour limiter les effets de bordure, la parcelle d'essai a été ceinturée par une garde de $3 \mathrm{~m}$ de largeur et de $30 \mathrm{~m}$ de longueur constituée par une variété commerciale (R579).

Variables quantitatives et qualitatives observées : Les variables agro-morphologiques, phytosanitaires et technologiques observées en cours de végétations pour présélectionner les clones au stade ligne étaient les suivantes: nombre de tiges usinables, diamètre de tige usinable, port végétatif, longueur de tige usinable, taux de floraison, présence de maladies endémiques (charbon, pokkah boeng ou échaudure foliaire), attaque sévère du foreur de tiges Eldana saccharina, brix (teneur en matière sèche dissoute dans le jus de canne). Ces observations qui permettaient de déterminer la vigueur végétative des clones ont donné lieu à une note globale variant de 0 à 4 pour chacun 
des individus testés, en comparaison avec la variété témoin. Le barème de notation était le suivant :

0 à 1 : Variétés à mauvaises aptitudes qui sont à éliminer ;

1,5: Variétés à repêcher, moins pourvues en certaines aptitudes ;

2 à 4 : Variétés à bonnes aptitudes $(\underline{3}$ : très bonnes aptitudes ; 4 : aptitudes exceptionnelles).

Les 148 variétés présélectionnées ont obtenu chacune une note globale supérieure à 2 et étaient indemnes de symptômes de maladies endémiques (échaudure foliaire, charbon, pokkah boeng). À 10 mois d'âge, les variables quantitatives suivantes ont été observées pour étudier la diversité de ces variétés : nombre de tiges usinables par ligne de $3 \mathrm{~m}$, diamètre de tige, longueur de tige usinable, taux de floraison et brix. Les variables qualitatives observées concernaient le tallage et le port végétatif.

Tableau 1. Caractères agro-morphologiques et technologiques ayant prévalu dans la pré-sélection des meilleurs clones au stade ligne. [Agro-morphological and technological traits used in preselection of best clones at one-row screening stage]

\begin{tabular}{l|l}
\hline Caractères & Méthodes d'observation \\
\hline $\begin{array}{l}\text { Agro-morphologiques } \\
\text { Nombre de tiges } \\
\text { usinables/3m }\end{array}$ & Comptage du nombre de tiges usinables à 10 mois. \\
\hline Tallage & Densité des talles observée à 3-4 mois (faible, moyen, très bon) \\
\hline Port végétatif & Appréciation de l'architecture des cannes usinables à 8 mois. \\
\hline Diamètre $(\mathrm{mm})$ & $\begin{array}{l}\text { Mesure du diamètre de l'entre-nœud médian sur un échantillon représentatif de 10 } \\
\text { cannes usinables à l'aide d'un pied à coulisse. }\end{array}$ \\
\hline Hauteur (m) & Mesure de la longueur moyenne d'un échantillon de 10 cannes usinables à 10 mois. \\
\hline \% Floraison & $\begin{array}{l}\text { Comptage du nombre de cannes ayant fleuri par rapport au nombre total de cannes } \\
\text { par ligne à 10 mois. }\end{array}$ \\
\hline Port végétatif & Notation de l'architecture des tiges par rapport à la verticale. \\
\hline $\begin{array}{l}\text { Phytosanitaires } \\
\text { Maladies et ravageur } \\
\text { endémiques }\end{array}$ & $\begin{array}{l}\text { Comptage de fouets charbonneux, de souches attaquées par l'échaudure foliaire et } \\
\text { le pokkha boeng. Notation des attaques sèvères d'E. saccharina. }\end{array}$ \\
\hline $\begin{array}{l}\text { Technologique } \\
\text { Brix }\end{array}$ & $\begin{array}{l}\text { Mesure au champ, à 10 mois, du taux de matière sèche dissoute dans le jus de } \\
\text { canne à l'aide d'un refractomètre portable. }\end{array}$ \\
\hline
\end{tabular}

Analyses statistiques : Les logiciels Excel, Statistica version 7.1 et $R 2.2$ ont été utilisés pour l'analyse des données. La méthode d'analyse adoptée est basée sur la caractérisation des clones selon les caractères mesurés. Pour ce faire, les données obtenues ont dans un premier temps été saisies à l'aide du logiciel Excel sous la forme d'une base de données d'où un tableau croisé dynamique (TCD) a été réalisé. Celui-ci a permis d'extraire de ladite base de données les variables pertinentes à analyser qui étaient les suivantes: tallage, diamètre, longueur de tige, port végétatif, taux de floraison, brix). Les logiciels Statistica et $\mathrm{R}$ ont par la suite été utilisés pour réaliser successivement trois méthodes d'analyses multivariées: analyse en composantes principales (ACP), classification ascendante hiérarchique (CAH) et analyse factorielle discriminante (AFD). Les données mesurées n'étant pas exprimées dans les mêmes échelles de mesure, il était nécessaire de les transformer en variables centrées réduites comme rapporté par (Benzécri, 1970). La Classification Ascendante Hiérarchique $(\mathrm{CAH})$ a ensuite été réalisée sur la base des distances euclidiennes entre les clones suivant la méthode de Ward comme option d'agrégation. Cette analyse multivariée a permis de structurer les variétés de canne présélectionnées selon leurs caractéristiques agromorphologiques et technologiques. L'AFD a été réalisée enfin pour évaluer à l'aide du test $\lambda$-Wilks au seuil de $5 \%$ la robustesse des groupes précédemment formés à partir de la $\mathrm{CAH}$. Elle permet de vérifier l'appartenance des individus aux groupes précédemment constitués à l'aide de la CAH (Volle, 1997). 


\section{RÉSULTATS}

Dispersion des variables quantitatives observées à la récolte : Le taux de floraison et le nombre de tiges usinables par ligne de $3 \mathrm{~m}$ ont été les caractères quantitatifs les plus dispersés avec des coefficients de variation de 86 et $22 \%$ respectivement (tableau 2). Cela suggère que ces variables ont contribué fortement

dans la diversité génétique des variétés étudiées. Les trois autres variables quantitatives observées (diamètre de tige, longueur de tige et brix réfractomérique) présentaient chacune une dispersion d'environ $10 \%$. Celles-ci devraient contribuer à un degré moindre à la diversité génétique de ces variétés.

Tableau 2: Moyennes des variables agro-morphologiques et technologiques observées à la récolte sur 148 variétés de canne d'origine $\mathrm{RCl}$ et leurs dispersions respectives. [Means of agro-morphological and technological traits observed at harvest on $148 \mathrm{RCl}$ varieties and their respective dispersion]

\begin{tabular}{l|c|c|c|c}
\hline & Moyenne & Minimum & Maximum & CV (\%) \\
\hline Nb_tiges/3m & 59 & 32 & 100 & 22,6 \\
Diamètre (mm) & 24,9 & 18,7 & 31,7 & 10,9 \\
Hauteur $(\mathrm{m})$ & 2,7 & 2,1 & 3,6 & 10,8 \\
Brix & 20,5 & 14,2 & 24,4 & 8,7 \\
\%Floraison & 34,5 & 0,0 & 94,4 & 86,0 \\
\hline
\end{tabular}

Analyse en composantes principales : Les trois couples de variables les mieux corrélés étaient Diamètre-Nombre de tiges, Brix-Nombre de tiges et \% Floraison-Brix, avec des coefficients de $-0.78,-0.58$ et 0.59 (tableau 3). Les plus faiblement corrélés étaient, par ordre croissant en valeur absolue, \%FloraisonDiamètre $(-0.08)$, Brix-Longueur de tiges $(0.22)$, Brixdiamètre $(-0.26)$, et Longueur de tiges-Diamètre $(0.31)$.
II ressort de l'ACP que les variables permettant de mieux expliquer la diversité des variétés présélectionnées sont, dans l'ordre décroissant, le taux de floraison, le nombre de tiges $/ 3 \mathrm{~m}$, le diamètre des tiges et la longueur des tiges. Le Brix est par contre la variable quantitative expliquant le moins la diversité des clones présélectionnés (figures 1 et 2 , tableau 4).

Tableau 3: Matrice de corrélations entre les caractères quantitatifs observés à la récolte chez les 148 variétés présélectionnées au stade ligne à Ferké. [Correlation matrix of quantitative traits observed at harvest on 148 preselected $\mathrm{RCl}$ varieties at one-row stage in Ferké]

\begin{tabular}{l|c|c|c|c|c}
\hline & Nb_tiges.3m & Diamètre & Hauteur & Brix & \%Floraison \\
\hline Nb_tiges/3m & 1,00 & & & & \\
Diamètre & $-\mathbf{0 , 7 8}$ & 1,00 & & & \\
Hauteur & $-0,49$ & 0,31 & 1,00 & & \\
Brix & $\mathbf{0 , 5 9}$ & $-0,26$ & 0,22 & 1,00 & \\
\%Floraison & $-0,48$ & $-0,08$ & 0,47 & $\mathbf{- 0 , 5 8}$ & 1,00 \\
\hline
\end{tabular}

En gras, les coefficients de corrélation supérieurs à 0,5 en valeur absolue. 


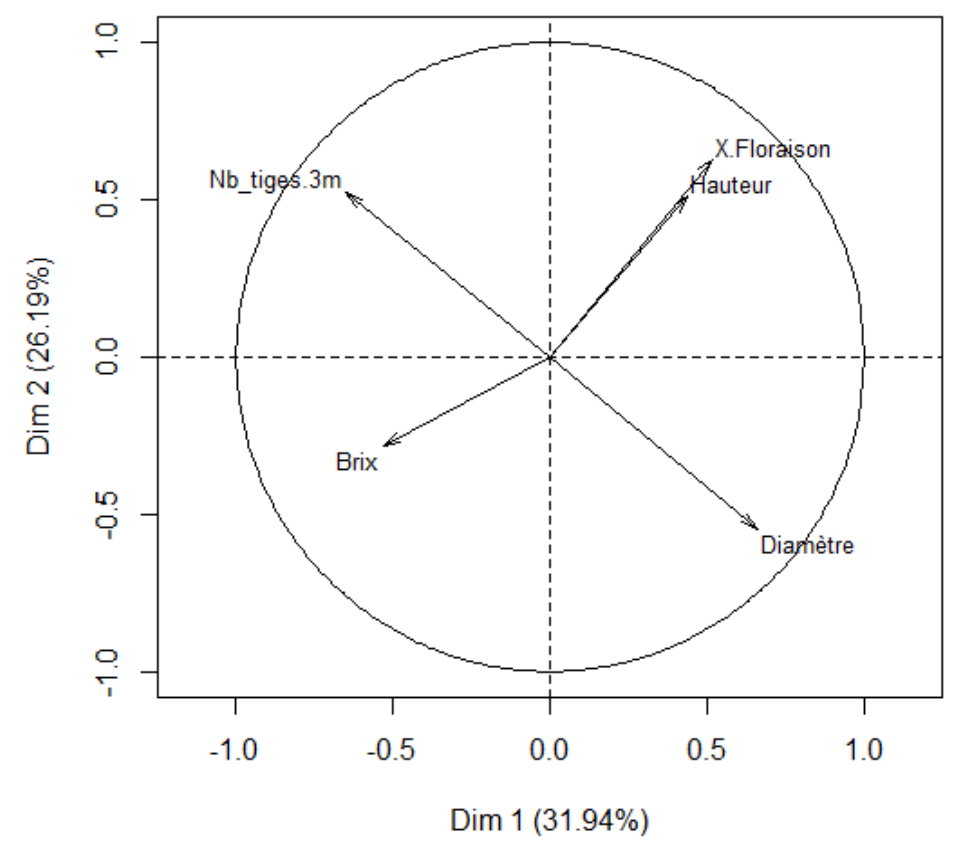

Figure 1: Cercle de corrélations résultant de la projection des variétés de cannes d'origine $\mathrm{RCl}$ et des variables agro-morphologiques et technologiques observées dans le plan factoriel 1-2 selon l'ACP. [Correlation circle resulting from projection of $\mathrm{RCl}$ varieties and agro-morphological traits observed in 1-2 factor plan following PCA]

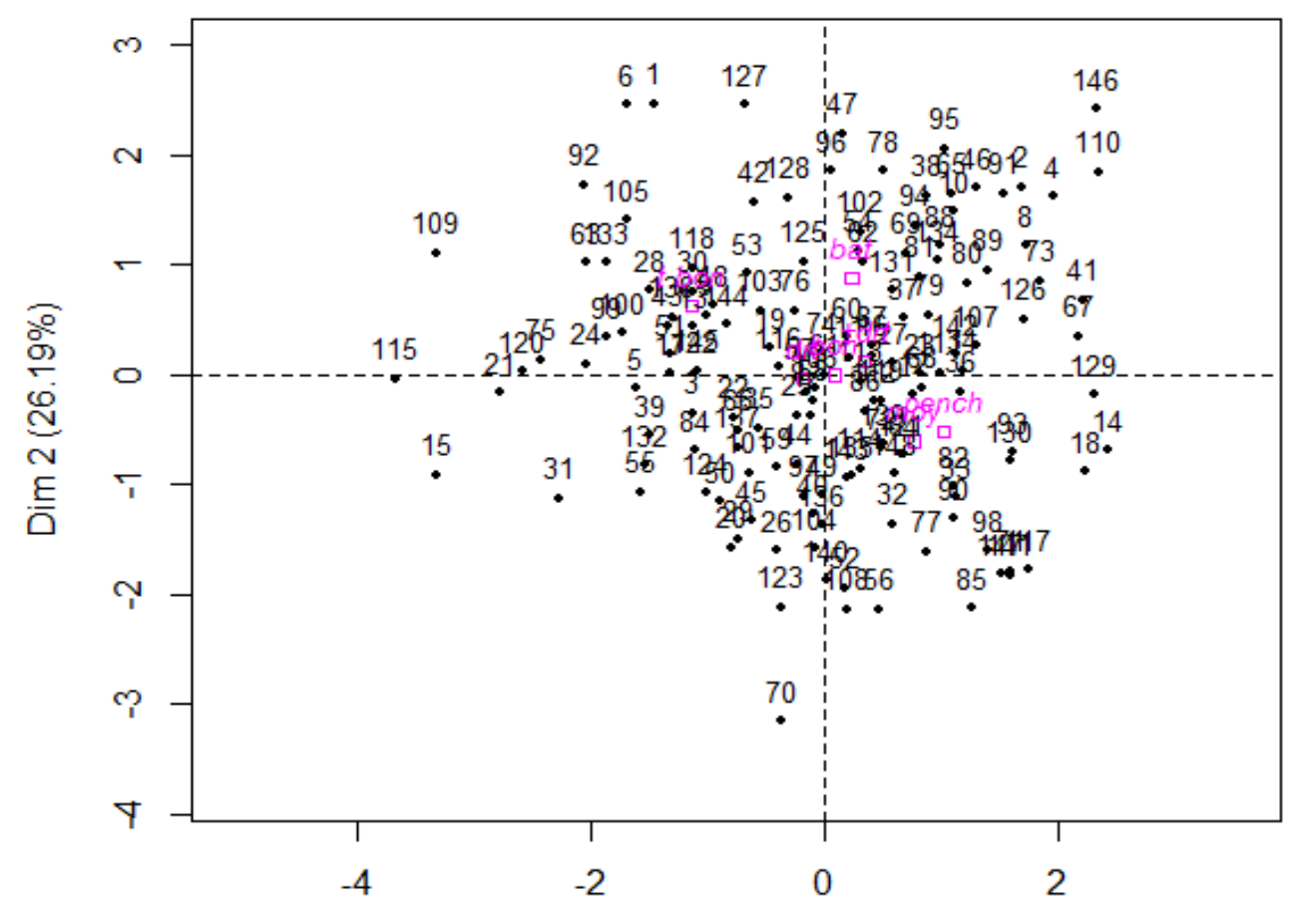

$\operatorname{Dim} 1(31.94 \%)$ 

Réunionno-Ivoirienne Présélectionnées à Ferké en Côte d'Ivoire

Figure 2. Projection des 148 variétés présélectionnées d'origine $\mathrm{RCl}$ et des variables qualitatives dans le plan factoriel 1-2 selon l'ACP basée la variance des caractères quantitatifs observés à la récolte. [Projection of $R C l$ cane varieties and qualitative traits in 1-2 factor plan following PCA based on quantitative traits observed at harvest]

Classification ascendante hiérarchique et analyse factorielle discriminante : Le dendrogramme résultant de la classification ascendante hiérarchique (CAH) basée sur la méthode de Ward, met en évidence 8 groupes de variétés pour un niveau de troncature égal à 0.16 ou $16 \%$ (figure 2). Cela suggère une assez bonne diversité génétique au sein des 148 individus présélectionnés. L'analyse factorielle discriminante (AFD) montre que ces 8 groupes de variétés sont significativement dissemblables $\quad(p<0.000001)$, (tableaux 5 et 6 ). Le tableau 7 présente le nombre et la liste nominative des variétés composant chaque groupe déterminé. Les groupes G4, G6, G1 et G3 sont les mieux représentés $(63.5 \%$ de l'effectif total) avec respectivement 30, 23, 21 et 20 individus. Les groupes G7, G5, G8 et G2 restants sont les moins représentés $(36.5 \%)$ avec respectivement $15,15,13$ et 11 individus. Les moyennes et les écarts-types des différents groupes pour chacune des variables quantitatives observées à la récolte sont données dans le tableau 8. Celui-ci montre que les variétés du groupe G1 ont le plus fort tallage tandis que celles de G7 se distinguent par leur faible tallage, avec en moyenne respectivement 75 et 47 tiges $/ 4.5 \mathrm{~m}^{2}$ soit environ 167000 et 104000 tiges/ha. Les variétés à fort taux de floraison sont issues du groupe $\mathrm{G} 8$ et celles à faible taux de G3, avec respectivement 70 et $4 \%$ en moyenne. Toutefois, ce faible taux de floraison est particulièrement variable chez les variétés du groupe G3 avec un coefficient de $138 \%$, contre $33 \%$ pour celles à fort taux (G8). Les autres groupes de variétés pour lesquels ce caractère est très variable (environ $100 \%$ ) sont G4 et G1. Les variétés potentiellement les plus riches en sucre sont issues des groupes G7, G2 et G1 avec en moyenne 22 de brix. Les variétés à longues tiges sont issues des groupes $G 5$ et $G 7$ et celles à tiges les plus courtes de $\mathrm{G} 1$ avec en moyenne, respectivement, environ 3 et $2.4 \mathrm{~m}$. II ressort de l'analyse factorielle des correspondances (AFC) que les variétés du groupe $\mathrm{G} 1$ ont un très bon tallage, variable réputée être associée à un bon rendement en canne et une bonne tenue en repousse (figure 3 ). Celles du groupe $\mathrm{G} 7$ ont un tallage moyen et un port végétatif plus ou moins droit. Les variétés du groupe $\mathrm{G} 2$ tendent à présenter un port droit qui est un caractère qualitatif recherché en récolte mécanisée de la canne à sucre comme cela est pratiqué en partie dans les complexes sucriers de Ferké, depuis une dizaine d'années, sur environ $30 \%$ des surfaces irriguées. Les ports penché et en bataille qui ont fortement contribué dans la répartition des différents groupes semblent être associés à 6 groupes à la fois (G2, G3, G4, G5, G6, G8). Sur les 148 variétés présélectionnées, les familles F62 et F31 étaient les plus représentées avec respectivement 36 et 18 individus. Ces deux familles sont également les plus représentées dans le groupe G4 avec respectivement 11 variétés/36 (30.5\%) et 9 variétés/18 $(50 \%)$. La famille $\mathrm{F} 62$ est encore bien représentée dans le groupe $\mathrm{G} 3$ avec 12 variétés/36 soit un taux de $33 \%$ (tableau 9). De plus, cette famille F62 s'avère la plus diversifiée par sa représentation dans 7 groupes de variétés (G4, G3, G6, G7, G1, G2 et G5). Elle est suivie par les familles F59 et F29 avec chacune 6 groupes d'individus. La famille prolixe F31 intervient en 3ème position avec 5 groupes de variétés (G4, G1, G3, G5 et G6).

Parmi les 8 groupes déterminés, G6 semble le plus diversifié génétiquement avec 16 familles différentes suivi de $\mathrm{G} 1$ avec 11 familles et de $\mathrm{G} 5$ avec 10 familles. Quant aux groupes G2 et G4, ils comportent chacun 8 familles. Les 2 groupes ayant le plus faible nombre de familles $(n=6)$ sont $G 3$ et $\mathrm{G} 8$. Les familles associées à un seul groupe de variétés, au nombre de 8 , sont F06, F07, F09, F27, F37, F44, F52 et F55. Celles associées à 2 groupes, au nombre de 8 aussi, sont F05, F02, F11, F15, F21, F24, F36, F58.

Tableau 4 : Numéros des variétés présélectionnées en 1ère repousse au stade ligne pour l'ACP.

\begin{tabular}{|c|c|c|c|c|c|c|c|c|c|}
\hline $\mathrm{N}^{\circ}$ & Variétés & $\mathrm{N}^{\circ}$ & Variétés & $\mathrm{N}^{\circ}$ & Variétés & $\mathrm{N}^{\circ}$ & Variétés & $\mathrm{N}^{\circ}$ & Variétés \\
\hline 1 & $\mathrm{RCl} 14 / 11$ & 31 & $\mathrm{RCl} 14 / 131$ & 61 & $\mathrm{RCl} / 14 / 161$ & 91 & $\mathrm{RCl12/191}$ & 121 & $\mathrm{RCl} 13 / 1121$ \\
\hline 2 & $\mathrm{RCl} 13 / 12$ & 32 & $\mathrm{RCl} 14 / 132$ & 62 & $\mathrm{RCl} 11 / 162$ & 92 & $\mathrm{RCl} 12 / 192$ & 122 & $\mathrm{RCl} 13 / 1122$ \\
\hline 3 & $\mathrm{RCl} 13 / 13$ & 33 & $\mathrm{RCl} 10 / 133$ & 63 & $\mathrm{RCl} 11 / 163$ & 93 & $\mathrm{RCl} 13 / 193$ & 123 & RCl13/1123 \\
\hline 4 & $\mathrm{RCl} 14 / 14$ & 34 & $\mathrm{RCl} 11 / 134$ & 64 & $\mathrm{RCl} 10 / 164$ & 94 & $\mathrm{RCl} 13 / 194$ & 124 & $\mathrm{RCl} 13 / 1124$ \\
\hline 5 & $\mathrm{RCl} 12 / 15$ & 35 & $\mathrm{RCl} 11 / 135$ & 65 & $\mathrm{RCl} 11 / 165$ & 95 & $\mathrm{RCl} 13 / 195$ & 125 & RCl14/1125 \\
\hline 6 & $\mathrm{RCl} 13 / 16$ & 36 & $\mathrm{RCl} 13 / 136$ & 66 & $\mathrm{RCl} 11 / 166$ & 96 & $\mathrm{RCl} 13 / 196$ & 126 & $\mathrm{RCl} 14 / 1126$ \\
\hline 7 & $\mathrm{RCl} 13 / 17$ & 37 & $\mathrm{RCl} 13 / 137$ & 671 & $\mathrm{RCl} 11 / 167$ & 97 & $\mathrm{RCl} 13 / 197$ & 127 & RCl14/1127 \\
\hline
\end{tabular}


Béhou et al, J. Appl. Biosci. 2018 Diversité Génétique des Premières Variétés de Canne à Sucre d'Origine Réunionno-Ivoirienne Présélectionnées à Ferké en Côte d'Ivoire

\begin{tabular}{|c|c|c|c|c|c|c|c|c|c|}
\hline 8 & $\mathrm{RCl} 14 / 18$ & 38 & $\mathrm{RCl} 13 / 138$ & 68 & $\mathrm{RCl} 11 / 168$ & 98 & $\mathrm{RCl13/198}$ & 128 & $\mathrm{RCl} 11 / 1128$ \\
\hline 9 & $\mathrm{RCl} 12 / 19$ & 39 & RCl13/139 & 69 & $\mathrm{RCl} 11 / 169$ & 99 & RCl14/199 & 129 & RCl11/1129 \\
\hline 10 & $\mathrm{RCl} 13 / 110$ & 40 & $\mathrm{RCl} 13 / 140$ & 70 & $\mathrm{RCl} 11 / 170$ & 100 & $\mathrm{RCl} 14 / 1100$ & 130 & RCl12/1130 \\
\hline 11 & $\mathrm{RCl} 14 / 111$ & 41 & RCl13/141 & 71 & RCI14/171 & 101 & RCl14/1101 & 131 & $\mathrm{RCl} 13 / 1131$ \\
\hline 12 & $\mathrm{RCl} 11 / 112$ & 42 & $\mathrm{RCl} 13 / 142$ & 72 & $\mathrm{RCl} 13 / 172$ & 102 & RCl14/1102 & 132 & RCl13/1132 \\
\hline 13 & $\mathrm{RCl} 11 / 113$ & 43 & $\mathrm{RCl} 13 / 143$ & 73 & $\mathrm{RCl} 13 / 173$ & 103 & $\mathrm{RCl} 14 / 1103$ & 133 & $\mathrm{RCl} 13 / 1133$ \\
\hline 14 & $\mathrm{RCl} 11 / 114$ & 44 & RCI13/144 & 74 & $\mathrm{RCl} 13 / 174$ & 104 & $\mathrm{RCl} 14 / 1104$ & 134 & RCl13/1134 \\
\hline 15 & $\mathrm{RCl} 11 / 115$ & 45 & $\mathrm{RCl} 13 / 145$ & 75 & $\mathrm{RCl} 13 / 175$ & 105 & $\mathrm{RCl} 14 / 1105$ & 135 & RCl13/1135 \\
\hline 16 & $\mathrm{RCl} 13 / 116$ & 46 & RCl14/146 & 76 & $\mathrm{RCl} 13 / 176$ & 106 & RCl14/1106 & 136 & $\mathrm{RCl} 13 / 1136$ \\
\hline 17 & $\mathrm{RCl} 13 / 117$ & 47 & RCl14/147 & 77 & $\mathrm{RCl} 13 / 177$ & 107 & RCl14/1107 & 137 & RCl14/1137 \\
\hline 18 & $\mathrm{RCl} 13 / 118$ & 48 & RCI14/148 & 78 & $\mathrm{RCl} 13 / 178$ & 108 & $\mathrm{RCl} 14 / 1108$ & 138 & RCl14/1138 \\
\hline 19 & $\mathrm{RCl} 13 / 119$ & 49 & $\mathrm{RCl} 12 / 149$ & 79 & $\mathrm{RCl} 13 / 179$ & 109 & RCl14/1109 & 139 & RCl14/1139 \\
\hline 20 & $\mathrm{RCl} 13 / 120$ & 50 & $\mathrm{RCl} 13 / 150$ & 80 & $\mathrm{RCl} 13 / 180$ & 110 & $\mathrm{RCl} 11 / 1110$ & 140 & RCl14/1140 \\
\hline 21 & $\mathrm{RCl} 13 / 121$ & 51 & $\mathrm{RCl} 13 / 151$ & 81 & $\mathrm{RCl} 13 / 181$ & 111 & RCl11/1111 & 141 & RCl14/1141 \\
\hline 22 & $\mathrm{RCl} 13 / 122$ & 52 & RCl13/152 & 82 & $\mathrm{RCl} 13 / 182$ & 112 & $\mathrm{RCl} 11 / 1112$ & 142 & RCl14/1142 \\
\hline 23 & $\mathrm{RCl} 13 / 123$ & 53 & $\mathrm{RCl} 13 / 153$ & 83 & $\mathrm{RCl} 13 / 183$ & 113 & $\mathrm{RCl} 11 / 1113$ & 143 & RCl14/1143 \\
\hline 24 & $\mathrm{RCl} 13 / 124$ & 54 & RCl14/154 & 84 & $\mathrm{RCl} 13 / 184$ & 114 & $\mathrm{RCl} 11 / 1114$ & 144 & RCl14/1144 \\
\hline 25 & $\mathrm{RCl} 13 / 125$ & 55 & RCI14/155 & 85 & $\mathrm{RCl} 13 / 185$ & 115 & RCl12/1115 & 145 & RCl14/1145 \\
\hline 26 & $\mathrm{RCl} 13 / 126$ & 56 & $\mathrm{RCl} 14 / 156$ & 86 & $\mathrm{RCl} 13 / 186$ & 116 & $\mathrm{RCl} 12 / 1116$ & 146 & RCl11/1146 \\
\hline 27 & $\mathrm{RCl} 14 / 127$ & 57 & $\mathrm{RCl} 14 / 157$ & 87 & $\mathrm{RCl} 13 / 187$ & 117 & $\mathrm{RCl} 13 / 1117$ & 147 & RCl14/1147 \\
\hline 28 & $\mathrm{RCl} 14 / 128$ & 58 & $\mathrm{RCl} 14 / 158$ & 88 & RCl14/188 & 118 & $\mathrm{RCl} 13 / 1118$ & 148 & RCl13/1148 \\
\hline 29 & $\mathrm{RCl} 14 / 129$ & 59 & RCI14/159 & 89 & RCI14/189 & 119 & $\mathrm{RCl} 13 / 1119$ & & \\
\hline 30 & $\mathrm{RCl} 14 / 130$ & 60 & $\mathrm{RCl} 14 / 160$ & 90 & $\mathrm{RCl} 11 / 190$ & 120 & $\mathrm{RCl} 13 / 1120$ & & \\
\hline
\end{tabular}

ACP : Analyse en Composantes Principales [Principal Component Analysis]

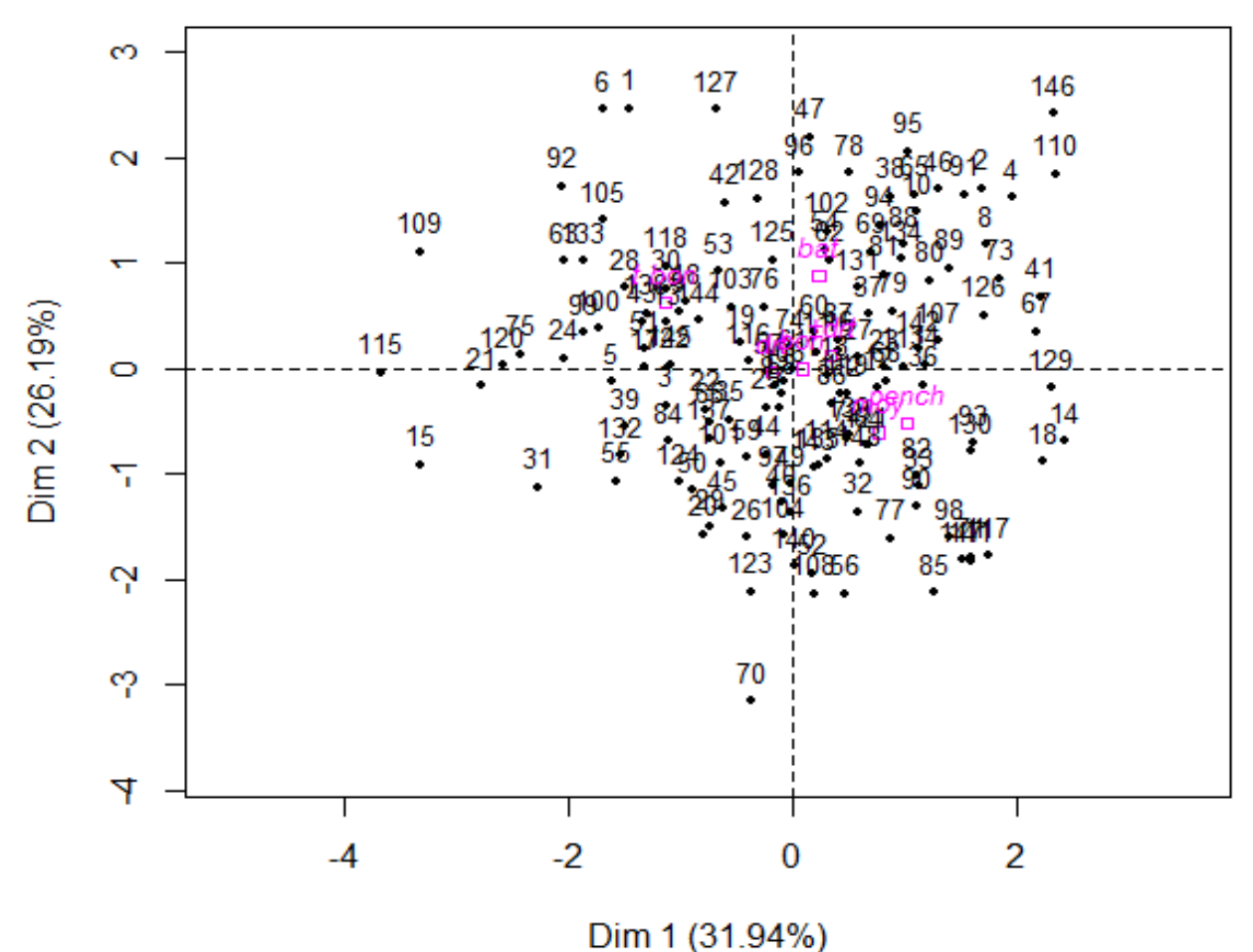

Figure 2 : Projection des 148 variétés présélectionnées d'origine $\mathrm{RCl}$ et des variables qualitatives dans le plan factoriel 1-2 selon l'ACP basée la variance des caractères quantitatifs observés à la récolte. [Projection of $R C l$ cane varieties and qualitative traits in 1-2 factor plan following PCA based on quantitative traits observed at harvest] 
Classification ascendante hiérarchique et analyse factorielle discriminante : Le dendrogramme résultant de la classification ascendante hiérarchique (CAH) basée sur la méthode de Ward, met en évidence 8 groupes de variétés pour un niveau de troncature égal à 0.16 ou $16 \%$ (figure 2). Cela suggère une assez bonne diversité génétique au sein des 148 individus présélectionnés. L'analyse factorielle discriminante (AFD) montre que ces 8 groupes de variétés sont significativement dissemblables $(p<0.000001)$, (tableaux 5 et 6 ). Le tableau 7 présente le nombre et la liste nominative des variétés composant chaque groupe déterminé. Les groupes G4, G6, G1 et G3 sont les mieux représentés ( $63.5 \%$ de l'effectif total) avec respectivement 30, 23, 21 et 20 individus. Les groupes G7, G5, G8 et G2 restants sont les moins représentés (36.5\%) avec respectivement $15,15,13$ et 11 individus. Les moyennes et les écarts-types des différents groupes pour chacune des variables quantitatives observées à la récolte sont données dans le tableau 8. Celui-ci montre que les variétés du groupe G1 ont le plus fort tallage tandis que celles de G7 se distinguent par leur faible tallage, avec en moyenne respectivement 75 et 47 tiges $/ 4.5 \mathrm{~m}^{2}$ soit environ 167000 et 104000 tiges/ha. Les variétés à fort taux de floraison sont issues du groupe $\mathrm{G} 8$ et celles à faible taux de G3, avec respectivement 70 et $4 \%$ en moyenne. Toutefois, ce faible taux de floraison est particulièrement variable chez les variétés du groupe G3 avec un coefficient de $138 \%$, contre $33 \%$ pour celles à fort taux (G8). Les autres groupes de variétés pour lesquels ce caractère est très variable (environ $100 \%$ ) sont $\mathrm{G} 4$ et $\mathrm{G} 1$. Les variétés potentiellement les plus riches en sucre sont issues des groupes G7, G2 et $\mathrm{G} 1$ avec en moyenne 22 de brix. Les variétés à longues tiges sont issues des groupes $\mathrm{G} 5$ et $\mathrm{G} 7$ et celles à tiges les plus courtes de $\mathrm{G} 1$ avec en moyenne, respectivement, environ 3 et $2.4 \mathrm{~m}$. II ressort de l'analyse factorielle des correspondances (AFC) que les variétés du groupe $\mathrm{G} 1$ ont un très bon tallage, variable réputée être associée à un bon rendement en canne et une bonne tenue en repousse (figure 3). Celles du groupe $\mathrm{G} 7$ ont un tallage moyen et un port végétatif plus ou moins droit. Les variétés du groupe $\mathrm{G} 2$ tendent à présenter un port droit qui est un caractère qualitatif recherché en récolte mécanisée de la canne à sucre comme cela est pratiqué en partie dans les complexes sucriers de Ferké, depuis une dizaine d'années, sur environ $30 \%$ des surfaces irriguées. Les ports penché et en bataille qui ont fortement contribué dans la répartition des différents groupes semblent être associés à 6 groupes à la fois (G2, G3, G4, G5, G6, G8). Sur les 148 variétés présélectionnées, les familles F62 et F31 étaient les plus représentées avec respectivement 36 et 18 individus. Ces deux familles sont également les plus représentées dans le groupe G4 avec respectivement 11 variétés/36 (30.5\%) et 9 variétés/18 (50\%). La famille F62 est encore bien représentée dans le groupe $\mathrm{G} 3$ avec 12 variétés/36 soit un taux de $33 \%$ (tableau 9). De plus, cette famille F62 s'avère la plus diversifiée par sa représentation dans 7 groupes de variétés (G4, G3, G6, G7, G1, G2 et G5). Elle est suivie par les familles $F 59$ et $F 29$ avec chacune 6 groupes d'individus. La famille prolixe F31 intervient en 3 ème position avec 5 groupes de variétés (G4, G1, $\mathrm{G} 3, \mathrm{G} 5$ et G6). Parmi les 8 groupes déterminés, G6 semble le plus diversifié génétiquement avec 16 familles différentes suivi de $\mathrm{G} 1$ avec 11 familles et de G5 avec 10 familles. Quant aux groupes G2 et G4, ils comportent chacun 8 familles. Les 2 groupes ayant le plus faible nombre de familles $(n=6)$ sont $G 3$ et $G 8$. Les familles associées à un seul groupe de variétés, au nombre de 8, sont F06, F07, F09, F27, F37, F44, F52 et $F 55$. Celles associées à 2 groupes, au nombre de 8 aussi, sont F05, F02, F11, F15, F21, F24, F36, F58. 


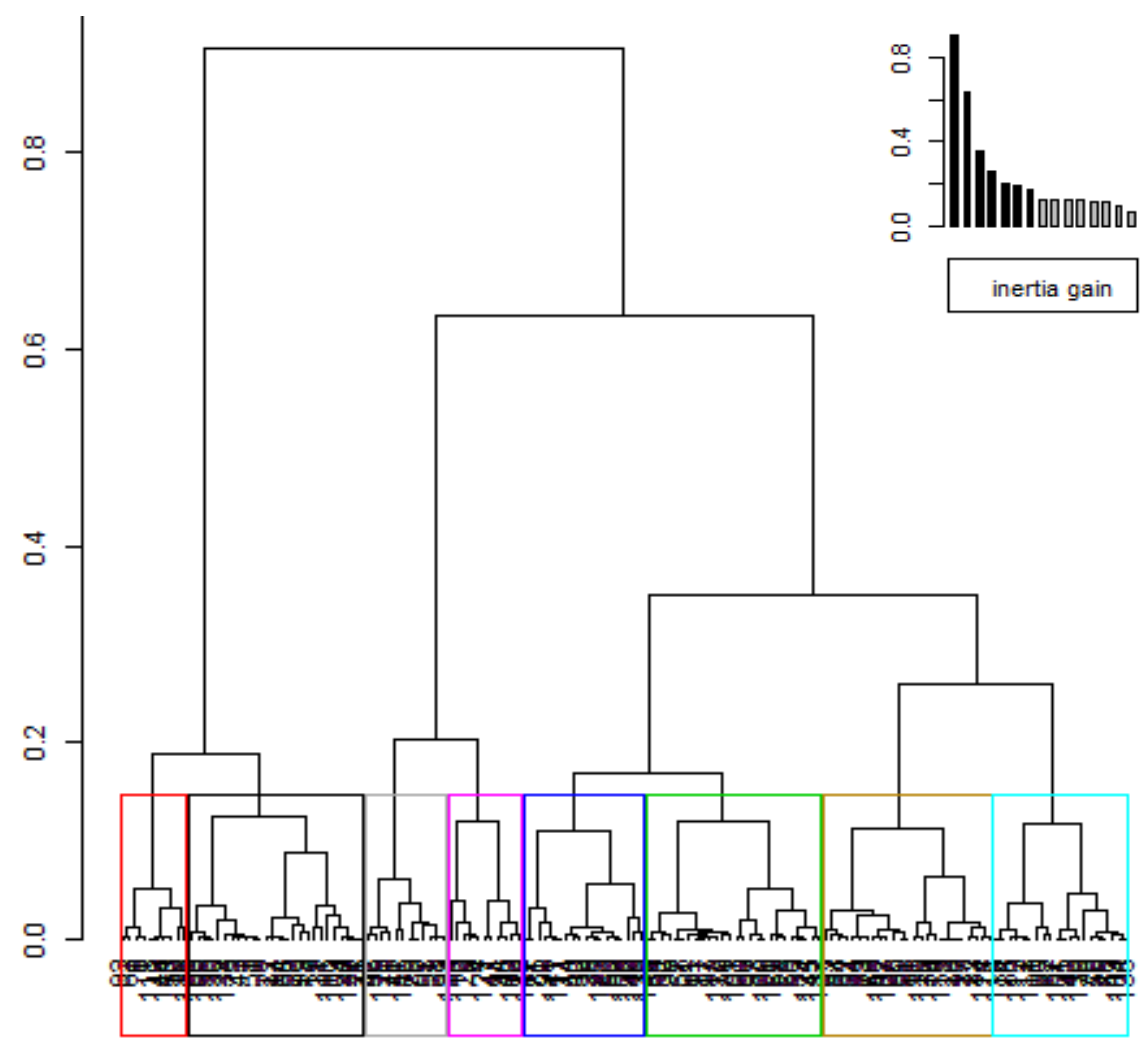

Figure 3: Dendrogramme résultant de la $\mathrm{CAH}$ relative aux 148 variétés d'origine $\mathrm{RCl}$ réparties en 8 groupes distincts. [Dendrogram resulting from cluster analysis regarding $148 \mathrm{RCl}$ cane varieties split into 8 different groups]

Tableau 5 : Implication des variables quantitatives observées dans la constitution des groupes de variétés déterminés.[involvement of quantitative traits observed in clustering of varieties]

\begin{tabular}{l|c|c|c|c|c|c}
\hline & Wilks (Lambda) & Partiel (Lambda) & F d'exc. (7,136) & Probab. (p) & Tolér. & 1-Tolér. ( $\left.\mathbf{R}^{2}\right)$ \\
\hline Nb_tiges.3m & 0,023503 & 0,660153 & 10,00183 & $\mathbf{0 , 0 0 0 0 0 0}$ & 0,973429 & 0,026571 \\
Diamètre & 0,027883 & 0,556457 & 15,48619 & $\mathbf{0 , 0 0 0 0 0 0}$ & 0,981933 & 0,018067 \\
Hauteur & 0,041463 & 0,374199 & 32,49181 & $\mathbf{0 , 0 0 0 0 0 0}$ & 0,981809 & 0,018191 \\
Brix & 0,035094 & 0,442120 & 24,51556 & $\mathbf{0 , 0 0 0 0 0 0}$ & 0,992174 & 0,007826 \\
\%Floraison & 0,042251 & 0,367219 & 33,47881 & $\mathbf{0 , 0 0 0 0 0 0}$ & 0,978525 & 0,021475 \\
\hline
\end{tabular}

Tableau 6 : Distance de Mahalanobis au carré (en dessous de la diagonale) entre les groupes de variétés pris deux à deux et valeurs de Fisher (au-dessus de la diagonale). [Mahalanobis distance square between clusters taken 2 by 2 and Fisher values]

\begin{tabular}{l|c|c|r|r|r|r|r|c}
\hline & Cluster 2 & Cluster 5 & \multicolumn{1}{c|}{ Cluster 3 } & Cluster 8 & Cluster 1 & Cluster 4 & Cluster 6 & Cluster 7 \\
\hline Cluster 2 & - & $\mathrm{F}=17,64$ & $\mathrm{~F}=21,87$ & $\mathrm{~F}=28,43$ & $\mathrm{~F}=17,92$ & $\mathrm{~F}=42,89$ & $\mathrm{~F}=18,22$ & $\mathrm{~F}=26,40$ \\
Cluster 5 & 14,30488 & - & $\mathrm{F}=23,70$ & $\mathrm{~F}=17,24$ & $\mathrm{~F}=48,73$ & $\mathrm{~F}=58,70$ & $\mathrm{~F}=17,06$ & $\mathrm{~F}=17,59$ \\
Cluster 3 & 15,86127 & 14,22889 & - & $\mathrm{F}=41,18$ & $\mathrm{~F}=18,51$ & $\mathrm{~F}=24,70$ & $\mathrm{~F}=26,52$ & $\mathrm{~F}=23,39$ \\
Cluster 8 & 24,55377 & 12,73791 & 26,90228 & - & $\mathrm{F}=52,27$ & $\mathrm{~F}=52,84$ & $\mathrm{~F}=15,56$ & $\mathrm{~F}=40,98$ \\
Cluster 1 & 12,77522 & 28,66366 & 9,30022 & 33,50342 & - & $\mathrm{F}=27,46$ & $\mathrm{~F}=34,64$ & $\mathrm{~F}=42,59$ \\
Cluster 4 & 27,42735 & 30,21162 & 10,59433 & 29,99069 & 11,44161 & - & $\mathrm{F}=25,26$ & $\mathrm{~F}=29,14$ \\
Cluster 6 & 12,60566 & 9,66919 & 12,75838 & 9,61582 & 16,24074 & 9,988444 & - & $\mathrm{F}=14,57$ \\
Cluster 7 & 21,41264 & 12,07119 & 14,04839 & 30,28743 & 25,05566 & 14,99903 & 8,25805 & - \\
\hline
\end{tabular}

$\mathrm{P}<0.00000$ pour toutes les valeurs de $\mathrm{F}$. 
Tableau 7 : Variétés de canne $\mathrm{RCI}$ présélectionnées composant les différents groupes déterminés par la $\mathrm{CAH}$. [RCI preselected cane varieties composing different clusters determined]

\begin{tabular}{|c|c|c|}
\hline $\begin{array}{l}N^{\circ} \\
\text { groupe }\end{array}$ & $\begin{array}{l}\text { Nombre de } \\
\text { variétés }\end{array}$ & Liste des variétés \\
\hline 1 & 21 & $\begin{array}{l}\text { RCl12/15; RCl11/113; RCl11/115; RCl13/117; RCl13/121; RCl13/122; } \\
\text { RCl13/124; RCl14/131; RCl13/139; RCl13/143; RCl14/155; RCl11/166; } \\
\text { RCl13/175; RCl13/184; RCl14/199; RCl14/1109; RCl12/1115; RCl13/1120; } \\
\text { RCl13/1122; RCl13/1132; RCl13/1133 }\end{array}$ \\
\hline 2 & 11 & $\begin{array}{l}\text { RCl14/11; RCl13/16; RCl13/119; RCl14/128; RCl14/148; RCl13/153; } \\
\text { RCl11/163; RCl12/192; RCl13/1118; RCl14/1125; RCl14/1144 }\end{array}$ \\
\hline 3 & 20 & $\begin{array}{l}\text { RCl13/13; RCl12/19; RCl13/125; RCl14/130; RCl11/135; RCl13/150; } \\
\text { RCl13/151; RCl14/157; RCl14/160; RCl14/161; RCl13/183; RCl13/197; } \\
\text { RCl14/1100; RCl14/1101; RCl14/1103; RCl14/1105; RCl14/1106; RCl14/1137; } \\
\text { RCl14/1138; RCl14/1145 }\end{array}$ \\
\hline 4 & 30 & $\begin{array}{l}\text { RCl13/17; RCl13/120; RCl13/126; RCl14/129; } \mathrm{RCl} 14 / 132 ; \mathrm{RCl} 13 / 140 ; \\
\mathrm{RCl} 13 / 144 ; \mathrm{RCl} 13 / 145 ; \mathrm{RCl} 12 / 149 ; \mathrm{RCl} 13 / 152 ; \mathrm{RCl} 14 / 156 ; \mathrm{RCl} 14 / 159 ; \\
\mathrm{RCl} 10 / 164 ; \mathrm{RCl} 11 / 170 ; \mathrm{RCl} 13 / 177 ; \mathrm{RCl} 13 / 182 ; \mathrm{RCl} 13 / 185 ; \mathrm{RCl} 13 / 198 ; \\
\mathrm{RCl} 14 / 1104 ; \mathrm{RCl} 14 / 1108 ; \mathrm{RCl} 11 / 1114 ; \mathrm{RCl} 13 / 1117 ; \mathrm{RCl} 13 / 1121 ; \mathrm{RCl} 13 / 1123 ; \\
\mathrm{RCl} 13 / 1124 ; \mathrm{RCl} 13 / 1135 ; \mathrm{RCl} 13 / 1136 ; \mathrm{RCl} 14 / 1140 ; \mathrm{RCl} 14 / 1141 ; \text { RCl13/1148 }\end{array}$ \\
\hline 5 & 15 & $\begin{array}{l}\mathrm{RCl} 13 / 12 ; \mathrm{RCl} 13 / 138 ; \mathrm{RCl} 13 / 142 ; \mathrm{RCl} 14 / 154 ; \mathrm{RCl} 11 / 162 ; \mathrm{RCl} 11 / 165 ; \\
\mathrm{RCl} 11 / 169 ; \mathrm{RCl} 13 / 178 ; \mathrm{RCl} 13 / 179 ; \mathrm{RCl} 13 / 180 ; \mathrm{RCl} 12 / 191 ; \mathrm{RCl} 13 / 195 ; \\
\mathrm{RCl} 13 / 196 ; \mathrm{RCl} 14 / 1102 ; \mathrm{RCl} 11 / 1128\end{array}$ \\
\hline 6 & 23 & $\begin{array}{l}\text { RCl14/111; RCl11/114; RCl13/116; RCl13/123; RCl14/127; RCl10/133; } \\
\text { RCl11/134; RCl13/136; RCl13/137; RCl14/158; RCl11/168; RCl13/172; } \\
\text { RCl13/173; RCl13/176; RCl13/181; RCl13/187; RCl14/188; RCl14/189; } \\
\text { RCl14/1107; RCl11/1113; RCl13/1119; RCl12/1130; RCl14/1142 }\end{array}$ \\
\hline 7 & 15 & $\begin{array}{l}\mathrm{RCl} 11 / 112 ; \mathrm{RCl} 13 / 118 ; \mathrm{RCl} 11 / 167 ; \mathrm{RCl} 14 / 171 ; \mathrm{RCl} 13 / 174 ; \mathrm{RCl} 13 / 186 ; \\
\mathrm{RCl} 11 / 190 ; \mathrm{RCl} 13 / 193 ; \mathrm{RCl} 11 / 1111 ; \mathrm{RCl} 11 / 1112 ; \mathrm{RCl} 12 / 1116 ; \mathrm{RCl} 11 / 1129 ; \\
\mathrm{RCl} 14 / 1139 ; \mathrm{RCl} 14 / 1143 ; \mathrm{RCl} 14 / 1147\end{array}$ \\
\hline 8 & 13 & $\begin{array}{l}\text { RCl14/14; RCl14/18; RCl13/110; RCl13/141; RCl14/146; RCl14/147; } \\
\text { RCl13/194; RCl11/1110; RCl14/1126; RCl14/1127; RCl13/1131; RCl13/1134; } \\
\text { RCl11/1146 }\end{array}$ \\
\hline
\end{tabular}

Tableau 8 : Moyennes des groupes de variétés RCI identifiés selon les variables quantitatives observées à la récolte en 1ère repousse. [Means of preselected $\mathrm{RCl}$ cane varieties following quantitative trait observed at harvest in ratoon 1]

\begin{tabular}{|c|c|c|c|c|c|c|c|c|c|c|}
\hline & \multicolumn{2}{|c|}{ Nb_tiges/3m } & \multicolumn{2}{|c|}{ Diamètre (mm) } & \multicolumn{2}{|c|}{ Hauteur (m) } & \multicolumn{2}{|l|}{ Brix } & \multicolumn{2}{|c|}{ \%Floraison } \\
\hline & Moy & CV (\%) & Moy & CV (\%) & Moy & CV (\%) & Moy & CV (\%) & Moy & CV (\%) \\
\hline Groupe 1 & 75 & 15.1 & 23.2 & 8.4 & 2.44 & 7.7 & 21.7 & 5.8 & 13.3 & 109.0 \\
\hline Groupe 2 & 66 & 17.1 & 20.7 & 6.8 & 2.65 & 7.5 & 22.0 & 4.3 & 61.4 & 21.0 \\
\hline Groupe 3 & 60 & 16.2 & 23.2 & 6.9 & 2.87 & 4.6 & 20.8 & 5.9 & 4.1 & 138.1 \\
\hline Groupe 4 & 49 & 16.3 & 30.0 & 6.4 & 2.49 & 7.1 & 20.2 & 6.7 & 11.6 & 106.8 \\
\hline Groupe 5 & 62 & 17.0 & 24.2 & 6.8 & 3.19 & 5.7 & 20.2 & 3.8 & 57.0 & 35.0 \\
\hline Groupe 6 & 53 & 13.8 & 26.1 & 7.5 & 2.71 & 5.6 & 19.8 & 3.8 & 56.7 & 31.8 \\
\hline Groupe 7 & 47 & 17.7 & 27.7 & 7.1 & 3.00 & 5.0 & 22.1 & 4.3 & 43.2 & 57.8 \\
\hline Groupe 8 & 64 & 19.4 & 25.0 & 8.0 & 2.83 & 7.7 & 16.9 & 8.3 & 70.0 & 33.2 \\
\hline
\end{tabular}




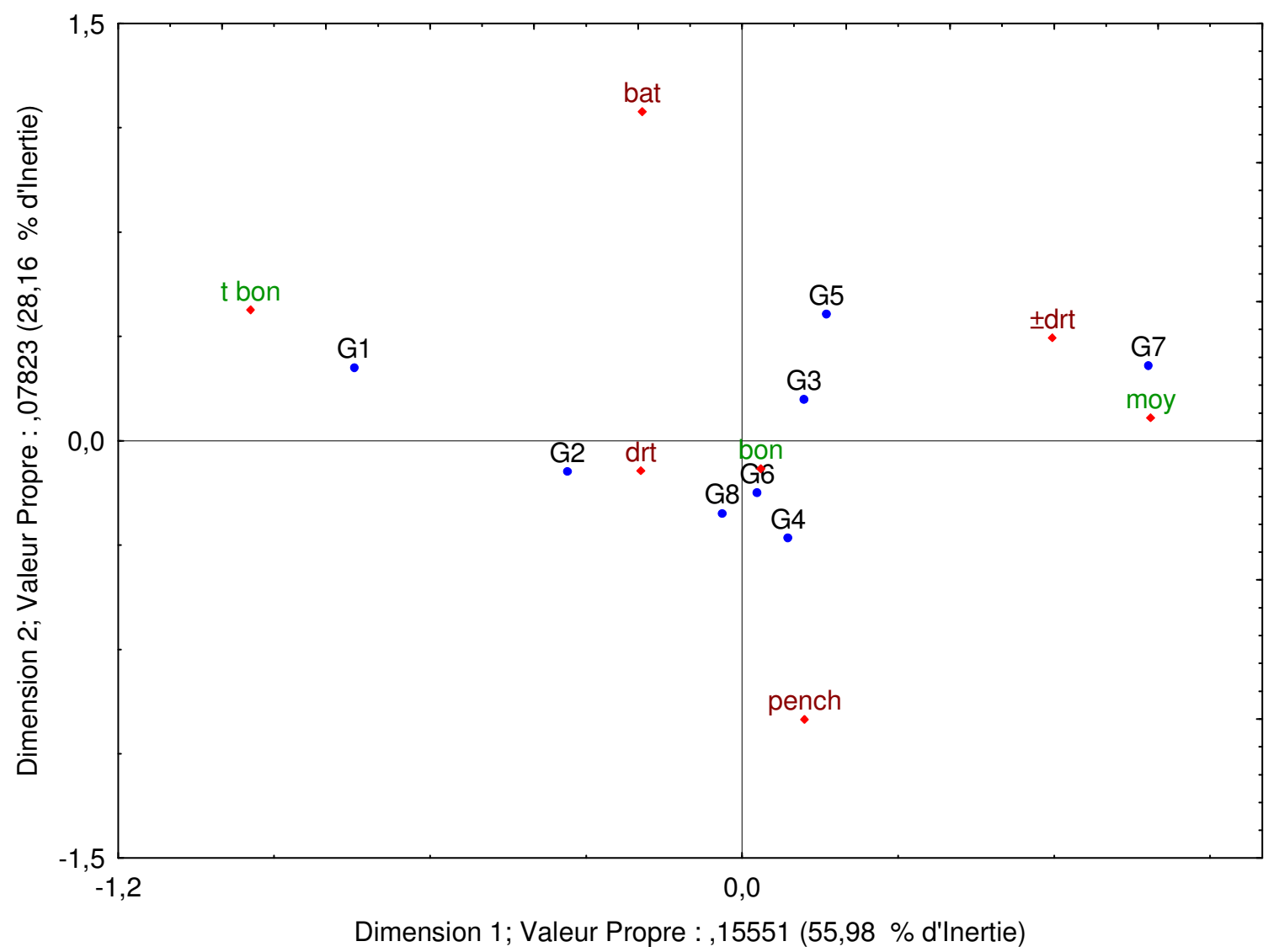

G1 : Groupe 1, G2 : Groupe 2, .., G8 : Groupe 8 ; $\mathbf{t}$ bon : tallage très bon, bon : tallage bon, moy : tallage moyen ; drt : port végétatif droit, \pm drt : port végétatif \pm droit, pench : port végétatif penché, bat : port végétatif en bataille.

Figure 4 : Projection des différents groupes formés et des caractères qualitatifs (tallage, port végétatif) dans le plan factoriel 1-2 selon l'AFC. [Projection of different clusters and qualitative traits in 1-2 factor plan following correspondence factor analysis]

Tableau 9 : Effectifs des variétés présélectionnées d'origine $\mathrm{RCl}$ selon les familles et les groupes discriminés par la classification ascendante hiérarchique $(\mathrm{CAH})$. [Total number of preselected $\mathrm{RCl}$ cane varieties following different families and clusters]

\begin{tabular}{l|c|c|c|c|c|c|c|c|c}
\hline & \multicolumn{1}{c}{ Groupes de variétés discriminés } \\
\hline Familles & G1 & G2 & G3 & G4 & G5 & G6 & G7 & G8 & Total \\
\hline NC0376 x N27 (F61) & 1 & 2 & - & - & 1 & 1 & - & - & 5 \\
R575 x Q140 (F36) & - & 1 & - & - & - & - & 1 & - & 2 \\
R579 x BT92/3586 (F31) & 5 & - & 2 & 9 & 1 & 1 & - & - & 18 \\
R579 x R585 (F62) & 3 & 2 & 12 & 11 & 1 & 4 & 3 & - & 36 \\
R579 x R97/0434 (F37) & - & - & - & - & - & 1 & - & - & 1 \\
R582 x R01/6043 (F27) & - & - & - & - & - & 1 & - & - & 1 \\
R582 x R570 (F52) & - & - & - & - & - & 1 & - & - & 1 \\
R582 x SP70/1143 (F58) & - & - & - & - & - & 1 & - & 1 & 2 \\
R89/2042 x R97/2332 (F11) & - & - & - & - & - & 2 & - & - & 2 \\
R92/2210 x R91/2069 (F24) & - & 1 & - & - & - & 1 & - & - & 2 \\
R92/2401 x R 98/6092 (F13) & 1 & - & - & 2 & - & - & - & - & 3 \\
R92/6545 x R93/6683 (F09) & - & - & - & - & 1 & - & - & - & 1
\end{tabular}




\begin{tabular}{|c|c|c|c|c|c|c|c|c|c|}
\hline R93/0136 x N27 (F50) & 1 & 1 & 1 & 1 & - & - & - & - & 4 \\
\hline R93/4255 x CP81/1405 (F42) & 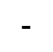 & - & - & - & 1 & - & 2 & ـ & 3 \\
\hline R93/6480 x FG04/517 (F63) & - & 1 & - & - & 2 & - & - & 2 & 5 \\
\hline R94/0142 x R98/6092 (F05) & - & - & - & - & - & 1 & 1 & - & 2 \\
\hline R94/6113 x R93/6885 (F04) & 1 & - & - & - & - & 1 & 3 & - & 5 \\
\hline R94/6113 x R97/6375 (F02) & - & - & - & 1 & - & 1 & - & - & 2 \\
\hline R96/0216 x R93/6776 (07) & - & - & 1 & - & - & - & - & - & 1 \\
\hline R 96/2116 x Q 213 (F32) & - & 1 & - & - & - & 1 & - & 6 & 8 \\
\hline R96/2569 x R585 (F59) & 2 & - & 3 & 2 & 2 & - & 1 & 1 & 11 \\
\hline R97/2335 x CP92/1641 (F30) & - & - & - & 2 & 4 & 1 & - & 1 & 8 \\
\hline R97/4004 x R95/4053 (F44) & - & - & - & - & 1 & - & - & - & 1 \\
\hline R97/6055 x R96/6422 (F21) & - & - & - & - & - & 2 & - & - & 2 \\
\hline $\mathrm{Rg9/2162} \mathrm{x} \mathrm{R01/2221} \mathrm{(F15)}$ & 1 & - & - & - & - & - & 1 & - & 2 \\
\hline R99/2162 x R585 (F56) & 2 & - & 1 & - & - & - & - & 2 & 5 \\
\hline SP70/3225 x R585 (F55) & - & - & - & - & - & - & 1 & - & 1 \\
\hline VMC93/282 x R01/6043 (F29) & 3 & 2 & - & 2 & 1 & 3 & 2 & - & 13 \\
\hline VMC93/282 x R97/2335 (F06) & 1 & - & - & - & - & - & - & - & 1 \\
\hline Total (29) & 21 & 11 & 20 & 30 & 15 & 23 & 15 & 13 & 148 \\
\hline
\end{tabular}

\section{DISCUSSION}

Variabilité des caractères quantitatifs : La forte dispersion du taux de floraison et du nombre de tiges usinables/3m, d'une part, et la faible dispersion $\mathrm{du}$ diamètre des tiges, de la hauteur des tiges et du brix observées chez les variétés présélectionnées à Ferké est en accord avec les travaux de Tadesse et al (2014) réalisés à Wonji en Éthiopie. La forte dispersion du nombre de tiges usinables/ha a été également rapportée par d'autres auteurs (Chaudhary, 2001); Balasundarum and Bhagyalakshmi, 1978 ; Nair et al, 1980 ; Sing et Sangwan, 1980). Selon Shivasubramanian and Menon (1973), les valeurs de coefficients de variation phénotypique (CVP) ou génotypique (CVG) sont classées de faibles de 0 et 10 $\%$, de moyennes de 11 à $20 \%$ et fortes au-delà de 20 $\%$ respectivement. Les travaux de Singh et al (1994) ont montré que des valeurs élevés de CVP et CVG traduisaient la pertinence des variables phénotypiques observées dans la sélection des variétés et suggéraient une bonne diversité génétique potentielle de celles-ci.

Groupes de variétés déterminés par la $\mathrm{CAH}$ : ॥ ressort que chacun des différents groupes de variétés déterminés par la classification ascendante hiérarchique (CAH) à partir des variables quantitatives observées est issu de plusieurs familles dont le nombre varie de 6 à 16. Cela montre que chaque groupe est assez diversifié génétiquement. Le nombre de groupes composant chaque famille de clones varie de 4 à 7 suggérant ainsi qu'elle est diversifiée phénotypiquement. La diversité relativement importante observée au sein de chaque groupe de variétés est conforme au fait que les géniteurs des familles étudiées couvrent jusqu'à 9 origines géographiques à travers le monde où la canne à sucre fait l'objet d'hybridations (AfrSud, Réunion, Australie, Floride, Brésil, Guadeloupe, Philippines, Barbade, Inde). Ces géniteurs sont eux-mêmes des variétés commerciales ou élites (hybrides hétérozygotes) qui sont issues d'hybridations dont les géniteurs couvrent également différentes origines géographiques. Les recombinaisons génétiques à travers ces hybridations permettent d'assurer une diversité variétale assez large pour prendre en compte la diversité des zones de culture de la canne à sucre ciblées, comme rapporté par différents auteurs (Tena et al, 2016 ; Singh et al, 1996 ; Das et al, 1996 ; Kumar et Ram, 1996). Elles se manifestent phénotypiquement par nombre de caractères agromorphologiques, technologiques ou phytosanitaires qui influencent le rendement en canne, la richesse en sucre et le taux d'extraction en sucre. Toutefois, différentes études ont montré que le nombre de groupes obtenus par la $\mathrm{CAH}$ pouvaient varier fortement selon les espèces, le nombre d'accessions et le type ou la quantité de variables observées (Brasileiro et al, 2014 ; Ortiz et al, 2008 ; Gonçalves et al, 2009 ; Barbé et al, 2010 ; Cabral, 2010). Les géniteurs utilisés dans les schémas de sélection ont été obtenus après plusieurs back-cross successifs (sélection récurrente) entre des clones de Saccharum officinarum et ceux d'espèces rustiques de Saccharum dont $S$. 
spontaneum pour la transmission du caractère richesse saccharine. Dans ce processus, le parent noble utilisé n'est pas nécessairement le même (D'Hont et al, 1998). Toutefois, ces croisements n'ont impliqué qu'un nombre restreint de clones sauvages. Cela suggère que seule une partie de la diversité génétique du genre Saccharum a été utilisée jusque-là dans la sélection de la canne à sucre. Cela conforte le fait que différentes études sur l'évaluation de la diversité génétique par l'utilisation de variables quantitatives et qualitatives, d'une part, et/ou de marqueurs moléculaires, d'autre part, ont révélé une forte similarité génétique entre des variétés différentes avec formation d'un nombre limité de groupes (Da silva et al, 2008 ; Oliveira et al, 2009; Duarte Filho et al, 2010; Dutra Filho et al, 2011; Sindhu et al, 2011; Santos et al, 2012; Santchurn et al, 2012; Perera et al, 2012). C'est pourquoi les schémas de sélection plus récents sont basés sur de nouveaux travaux de nobilisation de visant à introduire de la diversité supplémentaire dans le matériel végétal cultivé. Selon GOUY (2012), la sélection de la canne à sucre est récente car elle commence avec la création des premiers hybrides obtenus à Java au début du siècle dernier. La nobilisation, une méthode de croisements interspécifiques, permit l'obtention de variétés nettement plus performantes et résistantes aux principaux pathogènes de la canne. Depuis, les centres d'hybridations croisent des variétés élites et la sélection se fait à partir du phénotypage des descendances. Utiliser l'information moléculaire pour la sélection d'élites serait une réelle avancée pour le sélectionneur. Bien que de nombreuses études en génétique d'association soient réalisées, aucun marqueur n'est encore utilisé dans les schémas de sélection de la canne à sucre. Les effets des marqueurs ne sont pas estimés de façon assez précise et doivent être validés dans des populations indépendantes. L'association marqueurs-caractères est désormais complétée par de nouvelles approches telles que la sélection génomique et l'utilisation des méthodes éco-physiologiques dans la prévision du rendement.

Progrès génétique du rendement: Le progrès génétique est la différence positive entre la moyenne des descendants issus de reproducteurs sélectionnés et celle des descendants de reproducteurs choisis au hasard. Le progrès $(R)$, encore appelé gain génétique, dépend de l'intensité de la sélection (i), de l'héritabilité du caractère sélectionné $\left(h^{2}\right)$ et de l'écart-type phénotypique du caractère $\left(\sigma_{p}\right)$; il peut alors être calculé comme suit (Falconer, 1974) :

$R=i^{*} h^{2 *} \sigma_{p}$ $h^{2}=100 * \sigma_{g}^{2} / \sigma_{p}^{2}$ avec $\sigma_{g}^{2}$ : écart-type génotypique

Si l'intervalle entre générations de sélection est $t$, le progrès annuel s'écrit :

$\Delta G=\sigma_{p}{ }^{*}\left(i{ }^{*} h^{2}\right) / t$

Le progrès génétique annuel se définit alors comme le rapport entre le progrès génétique par génération (dans toute la population) et l'intervalle moyen entre générations. La FAO enregistre un rendement moyen mondial de la canne à sucre à 50,3 tha en 1961 et à 70,9 t/ha en 2009 (FAOSTAT, 2011). D'après ces données, les rendements ont donc connu une augmentation de $41 \%$ sur près de 50 ans, soit une moyenne de $0.8 \%$ de gain annuel. Ces augmentations sont dues à la fois à l'amélioration des pratiques agricoles et aux efforts de sélection. Un gain génétique moyen annuel de $0,6 \%$ a été observé à Barbade entre 1940 et 1975 (Simmonds, 1979). Hogarth estima à $1 \%$ l'augmentation annuelle moyenne du rendement au Queensland, en Australie (Hogarth, 1971). Une évaluation du gain génétique sur 33 ans d'expérimentations estime qu'environ $70 \%$ du gain enregistré s'expliquerait par les efforts de sélection (EDME et al, 2005). Cette étude montre également que les rendements en canne et en sucre n'ont pas encore atteint de plateau. En comparant des cultivars de différentes générations, il a été observé que les gains de rendements étaient principalement dus à une augmentation de la biomasse plutôt qu'à celle de la richesse saccharine, ceci étant plus prononcé à travers les cycles de culture (Jackson, 2005). L'auteur explique cela par l'insuffisance de pression de sélection sur le choix des géniteurs ainsi que sur leur descendance pour le caractère richesse saccharine. De plus, la diversité allélique pour ce caractère est étroite dans le germplasm cultivé. En effet, le nombre de croisements fondateurs est limité, soit environ une trentaine de parents communs, et les élites actuels sont principalement croisés entre eux. La sélection de la canne à sucre a permis une augmentation du rendement en sucre et un plateau ne semble pas avoir été atteint pour le rendement en canne (Edme et al, 2005 ; Jackson, 2005), ce qui laisse penser que des améliorations sont encore possibles. Des connaissances fines dans l'élaboration du rendement et les relations existantes entre ses composantes doivent être entreprises pour améliorer la sélection de ce caractère (Gouy, 2012).

Travaux en génétique quantitative sur le rendement: La génétique quantitative permet d'étudier la variabilité de caractères dans les populations et de connaître la part transmissible à la 
descendance. Jusqu'à une date récente, les études en la matière sur le rendement de la canne ont concerné principalement des variables telles que la biomasse, le tonnage de sucre/ha, la hauteur des tiges, leur diamètre et le tallage (Kang et al, 1989 ; Milligan et al, 1990 ; Sunil et Lawrence, 1996). Brown et al (1968) ont permis de donner un aperçu des héritabilités au sens strict, obtenues à partir de 47 croisements pour 24 caractères d'intérêt agronomique. Ces héritabilités restent faibles à moyennes avec des valeurs allant de 0,02 à 0,67 . Jackson étudia les composantes morphologiques et technologiques $\mathrm{du}$ rendement (nombre de talles $/ \mathrm{m}^{2}$, poids des talles, teneur en fibre, etc.) sur 141 individus des générations F1 et F2 issus de 32 croisements interspécifiques entre $S$. officinarun et $S$. spontaneum. Ces caractères présentent des héritabilités (au sens large) moyennes à fortes $(0,42$ à $0,75)$. Les études de génétique quantitative permettent de classer les caractères selon leur niveau d'héritabilité. II ressort principalement que la mesure de

\section{CONCLUSION ET APPLICATION DES RÉSULTATS}

L'étude a montré que les variables quantitatives permettant de mieux expliquer la diversité des variétés présélectionnées au stade ligne de $3 \mathrm{~m}$ comprenaient, dans l'ordre décroissant, le taux de floraison, le nombre de tiges $/ 3 \mathrm{~m}$, le diamètre des tiges et la longueur des tiges. Ces variables ont fortement contribué à la discrimination de 8 groupes qui suggère une assez bonne diversité génétique parmi les 148 clones de canne à sucre présélectionnés. Les 4 groupes les mieux représentés ( $63,5 \%$ de l'effectif total)

\section{RÉFÉRENCES CITÉES}

Balasundaram N, Bhagyalkshmi KV, 1978. Variability, heritability and association among yield and yield components in sugarcane. Indian $\mathrm{J}$. Agric. Sci. 48: 291-295.

Barbé TD, Gonçalves LSA, Rodrigues R, 2010. Association between advanced generations and genealogy in inbred lines of snap bean by the Ward-modified location model. Euphytica 173: 337-343.

Benzécri JP, 1970. Leçon sur l'analyse statistique des données multidimensionnelles. Faculté des sciences de paris.

Brasileiro BP, Marinho CD, Costa PMA, Moreira EFA, Peternelli LA, Barbosa MHP, 2014. Genetic diversity in sugarcane varieties in Brazil based on the Ward-Modified Location Model la richesse saccharine, notamment à travers le Brix, est un des caractères phénotypiques les plus héritables. Le tonnage de sucre/ha présente également une héritabilité plutôt élevée par rapport à celle des autres variables. À l'inverse, le tallage et le rendement en canne sont deux caractères à faibles héritabilités. Les corrélations génétiques obtenues pour les différentes études sont parfois contradictoires. Les résultats de Jackson (2005) montrent que d'un cycle à un autre, les corrélations génétiques peuvent s'inverser entre la richesse et le rendement en canne. Par contre, une corrélation positive entre le tallage et le rendement en canne se maintient sur les différentes repousses. Cette corrélation positive est également retrouvée dans l'étude de Sills qui travailla sur 44 descendants d'un croisement $S$. officinarun et $S$. spontaneum (SILLS et al, 1995). Jackson précise dans son étude que dans l'ensemble des recherches effectuées, peu de corrélations ont été observées.

comprenaient chacun 20 à 30 individus, tandis que les moins représentés (36.5\%) 11 à 15 individus chacun. Les variables qualitatives les plus pertinentes dans la discrimination des groupes concernaient très bon tallage, tallage moyen et port plus ou moins droit, d'une part, et port droit, d'autre part, qui sont associés, respectivement, aux groupes G1, G7 et G2. Quant aux variables port penché et port en bataille qui sont également pertinentes, elles semblaient être associées à 6 groupes à la fois (G2, G3, G4, G5, G6 et G8).

clustering strategy. Genet. Mol. Res. 13 (1) : 1650-1660.

Brown A, Daniels J, Latter B, Krishnamurthi M, 1968. Quantitative genetics of sugarcane. TAG (Theoret. and Applied Genet.) 39: 79-87.

Cabral PDS, Soares TCB, Gonçalves LSA, Amaral AT, 2010. Quantification of the diversity among common bean accessions using Ward-MLM strategy. Pesa. Agropec. Brasil 45: 11241132.

Chaudhary RR, 2001. Genetic variability and heritability in sugarcane. Nepal Agric. Res. J. Vol 4\&5: $\mathrm{xxX}-\mathrm{xxX}$.

Da silva CM, Mangolin CA, Mott AS, Machado MPS, 2008. Genetic diversity associated with in vitro and conventional bud propagation of 
Saccharum varieties using RAPD analysis. Plant Breed. 127: 160-165.

Das Pk, Nayak BC, Nayak N, Parida AK, 1996. Genetic variability in biometrical trials of early maturing genotypes of sugarcane. Indian Sugar 46: 723-726.

Duarte Filho L, Silva P, Santos J, Barbosa G, 2010. Genetic similarity among genotypes of sugarcane estimated by SSR and coefficient of parentage. Sugar Tech. 12: 145-149.

Falconer DS, 1974. Introduction à la génétique quantitative. Paris, Falconer (Ed). 250 p.

Gonçalves Ls, Rodrigues R, do Amaral Jr AT, Karasawa M, 2009. Heirloon tomato gene bank : assessing genetic divergence based on morphological, agronomic and molecular data using a Ward-modidied location model. Gent. Mol. Res. 8: 364-374.

Gouy M, 2012. Historique de l'amélioration de la canne à sucre et état de l'art des recherches en génétique d'association pour le rendement. In : Actes du Congrès sucrier AFCAS/ARTAS tenu en septembre 2012 à La Réunion. Paris, AFCAS/ARTAS, $300 \mathrm{p}$.

Hogarth D, 1971. Quantitative inheritance studies in sugarcane: II. Correlations and predicted responses to selection. Austral. J Agric. Res. 22: 103-109.

Jackson PA, 2005. Breeding for improved sugar content in sugarcane. Field Crops Res. 92: 277-290.

Kang M, Sosa O, Miller J, 1989. Path analysis for percent fiber and cane and sugar yield in sugarcane. Crop Sci. 29: 1481-1483.

Kouamé KD, Péné CB, Zouzou M, 2010. Évaluation de la résistance variétale de la canne à sucre au foreur de tiges tropical africain (Eldana saccharina Walker) en Côte d'Ivoire. J. of Applied Biosci. 26: 1614-1622.

Kumar S, Ram B, 1996. Genetic variability, heritability and correlations in hybrid $x$ species progenies of sugarcane grown under saline conditions. Sugarc. 2: 17-19.

Nair NV, Somarajan KG, Balasundaram N, 1980. Genetic variability, heritability and genetic advance in Saccharun officinarum. Int. Sugarc. J. XXXII : 275-276.

Oliveira KM, Pinto LR, Marconi TG, Mollinari M, 2009. Characterization of new polymorphic functional markers for sugarcane. Genome 52: 191-209.
Ortiz R, Sevilla R, Alvarado G, Crossa J, 2008. Numerical classification of related Peruvian highland maize races using internal ear traits. Genet. Resour. Crop. Ev. 55: 1055-1064.

Péné $\mathrm{CB}$, Déa $\mathrm{GB}, 2000$. Amélioration de la canne à sucre en Côte d'Ivoire. In : 1er atelier régional sur l'amélioration variétale de la canne à sucre en Afrique de l'Ouest et Centrale tenu en Octobre 1999 à Yamoussoukro. Montpellier, CIRAD : 98 - 102.

Péné CB, Kouamé DK, Dove H, Boua MB, 2016. Incidence des infestations du foreur de tiges Eldana saccharina (Lepidoptera, Pyralidea) en culture irriguée de canne à sucre selon la variété et la période de récolte en Côte d'Ivoire. J. of Applied Biosci. 102: 9687 9698.

Péné CB, Tuo K, 2007. Early and late-season screening of sugarcane varieties in north Ivory Coast, Annual report, 77 pages.

Perera MF, Arias ME, Costilla D, luque AC, 2012. Genetic diversity assessment and genotype identification in sugarcane based on DNA markers and morphologial traits. Euphytica 185: 491-510.

Santchurn D, Ramdoyal K, Badaloo MGH, Labuschagne M, 2012. From sugar industry to cane industry: investigations on multivariate data analysis technique in the identification of different high biomass sugarcane varieties. Euphytica 185: 543-558.

Santos JM, Durte Filho LSC, Soriano ML, Silva PP, 2012. Genetic diversity of the main progenitors of sugarcane from the RIDESA germplasm bank using SSR markers. Ind. Crop. Prod. 40: 145-150.

Shivasubramanian S, Menon M, 1973. Heterosis and inbreeding depression in rice. Madra Agric. J. 60: 1139

Sills GR, Bridges W, Al-Janabi SM, Sobral BWS, 1995. Genetic analysis of agronomic traits in a cross between sugarcane ( $S$. officinarum $L$.) and its presumed progenitor (S. robustum). Mol. Breed. 1: 355-363.

Simmonds NW, 1979. The impact of plant breeding on sugarcane yields in Barbados. Tropical Agric. Trinidad 56 (4) : 289-300.

Sindhu R, Govindaraj P, Balamurugan A, Appupnu C, 2011. Genetic diversity in sugarcane hybrids (Saccharum spp complex) grown in tropical 
India based on STMS markers. J. Plant Biochem. Biol. 20: 118-124.

Singh AP, Chatterjee A, Nema GK, 1996. Genetic variability in sugarcane (Saccharum spp. complex). Crop Res. (Hisar) 12: 162-164.

Singh R, Sangwan RS, 1980. Studies on genetic variability for stalk characters in sugarcane. Indian Sugar 30: 409-412.

Singh RK, Singh DN, Singh SK, Singh HN, 1994. Genetic variability and correlation studies in foreign commercial hybrids of sugarcane. Agric. Sci. Dig., Karnal : 103-107.

Sunil H, Lawrence M, 1996. Quantitative genetics of sugarcane. I. A large- scale evaluation of Saccharum germplasm. Sugar Cane (UK).

Tena E, Mekbib F, Ayana A, 2016. Genetic diversity of quantitative traits of sugarcane geneotypes in Ethiopia. Americ. J. Plant Sci. 7: 1498-1520.
Tadesse F, Negi T, Getaneh A, Dilnesaw Z, Ayele N, Teferi Y, 2014. Genetic variability and heritability of ten exotic sugar cane genotypes at Wonji Sugar Estate of Ethiopia. Global Advanced Research J. of Physical and Applied Sci. Vol 3(4) : XXX-XXX.

Volle M, 1997. L'analyse des données. In : Economie et statistique. Institut national de la statistique et des études économiques 96 (1) : 3-23.

Zadi M., Turquin L., Péné C. B., 2017. Évaluations agronomiques et technologiques des clones de canne à sucre (Saccharum officinarum L.) présélectionnés au stade ligne à Ferkessédougou, Nord Côte d'Ivoire. Journal of Applied Biosciences 115 : 11502 - 11512. 